\title{
Performance Analysis of Scheduling in Multiuser MIMO Systems with Zero-Forcing Receivers
}

\author{
Chiung-Jang Chen and Li-Chun Wang, Senior Member, IEEE
}

\begin{abstract}
Despite its low-complexity, the zero-forcing receiver is known to suffer from noise enhancement to restore the spatially multiplexed data in a single-user MIMO system. Nevertheless, in the multiuser system, the poor-channel avoidance property of the scheduling technique provides a natural way to overcome the drawback of noise enhancement [20]. In this paper, we present an analytical framework to evaluate the performance of the zero-forcing receiver operating in the multiuser MIMO system with user scheduling. Using the order statistics technique, we derive closed-form expressions for the sum-rate capacity of the multiuser MIMO system that employs the simple spatial multiplexing at the transmitter and zero-forcing processing at the receiver with a number of scheduling algorithms. These closed-form expressions hold for an arbitrary finite number of users and facilitate efficient numerical evaluations for cases of practical interest. In addition, the tractable analysis provides insight into how the scheduling technique affects the performance of the multiuser MIMO system under scalar feedback and vector feedback. The results are also extended to the case of heterogonous users with unequal average SNR.
\end{abstract}

Index Terms-MIMO systems, cross-layer cooperation, zeroforcing receiver, scheduling, fading channels.

\section{INTRODUCTION}

$\mathbf{U}$ TILIZING the multiple-input multiple-output (MIMO) antenna techniques to achieve high data rate transmission has attracted great attention since the pioneering works [1] and [2]. Subsequent research continues to explore the theoretical capacity limit of the MIMO channel under different assumptions concerning the spatial correlation and the available channel information [3]-[5]. Furthermore, a lot of efforts have been made to realize the promised capacity benefit by developing practical MIMO transmission schemes [6]. One popular approach is the spatial multiplexing method that sends parallel data streams across $N_{t}$ multiple transmit antennas [6], [7]. In case the channel exhibits rich scattering and the receiver has $N_{r}$ multiple antennas satisfying $N_{r} \geq N_{t}$, the spatially multiplexed signals can be successfully restored at the receiver, thereby providing about $N_{t}$ times higher capacity gain than the system equipped with only one transmit and one receive antenna.

Various receiver architectures, such as the zero-forcing receiver, the minimum mean square error (MMSE) receiver and the successive interference canceller (SIC), have been investigated to decode the spatially multiplexed signals over

\footnotetext{
Manuscript received Jun. 1, 2006; revised Dec. 24, 2006. This paper was presented in part at IEEE Globecom'05, St. Louis, Dec. 2005.

C.-J. Chen is with Chunghwa Telecom Laboratories, Taiwan, R.O.C. (email: cjch@cht.com.tw).

L.-C. Wang is with National Chiao Tung University, Taiwan, R.O.C. (email: lichun@cc.nctu.edu.tw).

Digital Object Identifier 10.1109/JSAC.2007.070916.
}

the MIMO system [7]-[10]. Generally speaking, the performance improvement from one type of the MIMO receiver to another usually comes at the price of higher implementation costs. For example, the zero-forcing receiver with simple independent decoding is known to suffer from the effect of noise enhancement despite its low complexity. On the other hand, the SIC-based receiver can possibly achieve the full capacity gain of the MIMO system with much higher complexity [8]. How to improve the performance of MIMO receivers while maintaining implementation complexity at a reasonable level still remains an important and open issue.

In a multiuser MIMO system, however, one can also exploit multiuser diversity to enhance the performance of the zeroforcing receiver by implementing scheduling algorithms [20], [21]. The excellence of engaging multiuser diversity with the zero-forcing receiver can be explained as follows. Consider a multiuser MIMO system where the base station with $N_{t}$ transmit antennas serves $K$ downlink users, each of which is equipped with $N_{r}$ receive antennas. Assume that each user employs the zero-forcing receiver to recover the spatially multiplexed data transmitted from the base station. Although the $N_{t} \times N_{r}$ MIMO link between the base station and any user could encounter noise enhancement under poor channel conditions, in a multiuser system with scheduling the base station can arrange transmissions for those users with better channel quality so that the impact of noise enhancement can be alleviated. In other words, the inherent property of poor-channel quality avoidance from the multiuser scheduling system provides a natural way to overcome the drawback of noise enhancement for the zero-forcing receiver. In this regard, employing the simple zero-forcing MIMO receiver on top of the multiuser scheduling system (such as IS-856 [11] and HSDPA [12]) results in a nice cross-layer synergy that can further improve the spectral efficiency in a practical and costeffective manner.

The multiuser MIMO downlink system under consideration is generally a MIMO broadcast channel (BC) from the viewpoint of information theory. Recently, the capacity region of MIMO BCs is derived in [13], [14] via the technique of uplink-downlink duality. The maximum sum-rate capacity of MIMO BCs can be achieved by a dirty paper coding (DPC) scheme that pre-subtracts interference from other users at the transmitter [15], [16]. While DPC is shown to be the optimal (capacity-achieving) strategy, its high computational burden and stringent feedback requirement motivate a research line of seeking other suboptimal and good-performance transmission strategies in MIMO BCs [17]-[23]. In particular, combining the simple zero-forcing algorithm with multiuser 
diversity has been shown to be a low-cost and effective way to achieve a large portion of MIMO BC capacity [18][21]. With a base station equipped with multiple antennas and each user with only single antenna, the zero-forcing beamforming method combined with proper user selection can gain a significant fraction of MIMO BC capacity [18], and is shown to be asymptotically optimal when the number of users $K$ approaches infinity [19]. When users have also multiple antennas, the suboptimal transmission strategy that employs the simple spatial multiplexing at the transmitter and zero-forcing processing at the receiver can also fulfill a large portion of MIMO BC capacity in the multiuser scheduling environment [20], [21]. The authors in [20] demonstrate via simulations that the performance of the suboptimal zeroforcing receiver approaches that of the optimal receiver under large $K$. Moreover, it is shown in [21] and [22] that when $K$ approaches infinity the capacity achieved by the simple zero-forcing receiver together with multiuser diversity scales identically to that of MIMO BCs achieved by the optimal DPC strategy. In addition, the authors in [23] proposed the random beamforming technique with the aim of reducing the amount of feedback. While [21] and [22] studied the transmission strategy of employing the scheduling technique and zeroforcing receiver in the regime of infinite user population, analytical characterization of the capacity gain achieved by such a transmission strategy in the pre-limit regime with finite users is also an important issue for realizing practical MIMO communications.

In this paper, similar to the setting of [20]-[22], we consider the suboptimal transmission strategy for MIMO broadcast channels that uses the simple spatial multiplexing at the transmitter and the zero-forcing processing at the receiver operating under a variety of scheduling algorithms. Using the order statistics technique, we are able to derive the probability distribution functions (PDFs) of effective subchannel output SNRs in the multiuser MIMO system with the various scheduling algorithms requiring scalar or vector feedback. Starting from these PDFs, we derive closed-form expressions, which hold for an arbitrary finite number of users and antennas, for the sum-rate capacity of the multiuser MIMO system. Specifically, we consider the max-max and max-min scheduling algorithms for the scalar feedback scheme and the spatially-independent scheduling algorithm for the vector feedback scheme. The scalar feedback means that each user only sends back one scalar value to indicate its channel condition, while the vector feedback provides the base station with more channel side information by allowing each user to feed back a vector of values to indicate all its subchannel output SNRs. Based on the analytical formulas and results, we characterize the capacity gain of the simple transmission strategy of combining the zeroforcing receiver and multiuser diversity and investigate the impact of the amount of feedback on the resulting performance. We also extend the results to the case of heterogenous users. The proposed analytical framework can be applied to other scheduling algorithms in addition to those considered in this paper.

We focus on the downlink multiuser MIMO system with $N_{r} \geq N_{t}$ and consider both the TDMA (time division multiple access) and space-time scheduling protocols in this work. The
TDMA scheduling protocol allows the base station to serve only one user at a time while simultaneously multiple users can be served for the space-time scheduling. Although the TDMA scheduling is strictly suboptimal for the multiuser MIMO system with $N_{r}<N_{t}$ from the viewpoint of achievable sum-rate, both the TDMA and space-time scheduling can achieve identical system multiplexing gain for $N_{r} \geq N_{t}$ [34], [35]. Since the space-time scheduling requires more channel side information of all users at the transmitter, the stringent feedback requirement and high computational complexity may appear challenging for practical implementation. In the scenario where the receivers have multiple antennas, using the TDMA scheduling provides the potential advantages of reducing feedback overhead and amortizing implementation complexity over multiple receivers without sacrificing system performance too much. The $2 \times 2$ antenna configuration has been adopted in contemporary industrial standards such as IEEE 802.16e [36] and 3GPP R7 [37].

The remaining parts of this paper are organized as follows. In Section II, we consider a single-user MIMO system and quantify the impact of noise enhancement for the zero-forcing receiver. In Section III, we describe the system model for the multiuser MIMO system with scheduling. Section IV analyzes the performance of the multiuser MIMO system with various scheduling policies. In Section V, we extend the scope of this paper to discuss the heterogeneous multiuser MIMO system wherein each user can have different average SNR. We give numerical results in Section VI and finally provide concluding remarks in Section VII.

\section{BACKGROUND AND MOTIVATION}

To begin with, we examine the performance of a singleuser spatial multiplexing MIMO system employing the zeroforcing receiver. We characterize the output SNR statistics of the zero-forcing receiver and then quantify the impact of noise enhancement on the maximum achievable throughput.

\section{A. The Zero-Forcing Receiver for MIMO Systems (Single- User Case)}

Consider a point-to-point MIMO system consisting of $N_{t}$ transmit and $N_{r}$ receive antennas. Let $\mathbf{x} \in \mathbb{C}^{N_{t} \times 1}$ and $\mathbf{y} \in$ $\mathbb{C}^{N_{r} \times 1}$ be the signal vectors at the transmitter and the receiver, respectively. Let $\mathbf{n} \in \mathbb{C}^{N_{r} \times 1}$ denote the spatially white noise with the covariance matrix $\mathbb{E}\left[\mathbf{n n}^{H}\right]=\sigma^{2} \mathbf{I}_{N_{r}}$, where $\mathbb{E}[\cdot]$ is the expectation operation and $(\cdot)^{H}$ is the transpose conjugate. Then, the link between $\mathbf{x}$ and $\mathbf{y}$ is modelled by

$$
\mathbf{y}=\mathbf{G} \mathbf{x}+\mathbf{n}=\sqrt{g} \mathbf{H} \mathbf{x}+\mathbf{n}
$$

where $\sqrt{g}$ depicts the large-scale (local average) behavior of the channel gain, and the normalized channel matrix $\mathbf{H} \in \mathbb{C}^{N_{r} \times N_{t}}$ characterizes the channel fading. We consider the flat Rayleigh fading such that every entry of $\mathbf{H}$ is an independent, circular-symmetric complex Gaussian random variable $\mathcal{C} \mathcal{N}(0,1)$. The total transmit power across all $N_{t}$ antennas is constrained by $\mathbb{E}\left[\mathbf{x}^{H} \mathbf{x}\right] \leq P_{T}$.

Assume that the spatial multiplexing with equal power allocation method is applied at the transmitter to send $N_{t}$ parallel 
data streams over the MIMO channel. Meanwhile, the suboptimal linear zero-forcing algorithm that simply inverts the channel and independently decodes the data streams is used at the receiver to recover the spatially multiplexed signals. By multiplying $\mathbf{y}$ with $\mathbf{H}^{\dagger} / \sqrt{g}$, where $\mathbf{H}^{\dagger}=\left(\mathbf{H}^{H} \mathbf{H}\right)^{-1} \mathbf{H}^{H}$ is the pseudo-inverse of $\mathbf{H}$, the received signal vector is expressed by

$$
\hat{\mathbf{x}}=\mathbf{H}^{\dagger} \mathbf{y} / \sqrt{g}=\mathbf{x}+\mathbf{H}^{\dagger} \mathbf{n} / \sqrt{g}
$$

Since the noise is colored by the $\mathbf{H}^{\dagger}$ operation, the components in $\hat{\mathbf{x}}$ are generally not independent. However, one can use a simple detection scheme that decodes each component of $\hat{\mathbf{x}}$ independently with reduced implementation complexity [20], [24]. With this simple independent decoding, the zeroforcing receiver decouples the MIMO channel into $N_{t}$ parallel channels. Moreover, the output SNR at the $n$-th subchannel after the zero-forcing processing can be expressed by

$$
\gamma_{n}=\frac{\rho}{N_{t}\left[\left(\mathbf{H}^{H} \mathbf{H}\right)^{-1}\right]_{n n}}, \quad n=1, \cdots, N_{t}
$$

where $[\mathbf{A}]_{i j}$ is the $(i, j)$-th entry of $\mathbf{A}$ and $\rho=P_{T} g / \sigma^{2}$ is the mean received SNR. It is shown in [24] that $\gamma_{n}$ in (3) is a Chisquared distributed random variable with degrees of freedom equal to $2\left(N_{r}-N_{t}+1\right)$. That is, the probability distribution function (PDF) of $\gamma_{n}$ can be written as

$$
f_{\gamma_{n}}\left(\gamma_{n}\right)=\frac{N_{t} e^{-N_{t} \gamma_{n} / \rho}}{\rho\left(N_{r}-N_{t}\right) !}\left(\frac{N_{t}}{\rho} \gamma_{n}\right)^{N_{r}-N_{t}}, \quad \gamma_{n} \geq 0 .
$$

Note that the assumption of $N_{r} \geq N_{t}$ has been implicitly made for the spatial multiplexing MIMO system. Next, we utilize (4) to derive the maximum achievable throughput of the zero-forcing receiver.

\section{B. Maximum Achievable Throughput}

With the subchannel output SNRs given in (4) from independent decoding, the maximum achievable throughput of the zero-forcing receiver can be calculated by summing up the throughput contributed from all subchannels. That is,

$$
\begin{aligned}
C_{\mathrm{zf}}= & \sum_{n=1}^{N_{t}} \mathbb{E}\left[\log \left(1+\gamma_{n}\right)\right]=N_{t} \int_{0}^{\infty} \log \left(1+\gamma_{n}\right) f_{\gamma_{n}}\left(\gamma_{n}\right) d \gamma_{n} \\
= & \frac{N_{t} e^{N_{t} / \rho} N_{t}^{N_{r}-N_{t}+1}}{\rho^{N_{r}-N_{t}+1}} \sum_{n=1}^{N_{r}-N_{t}+1}\left(\frac{\rho}{N_{t}}\right)^{n} \\
& \cdot \Gamma\left(n-N_{r}+N_{t}-1, \frac{N_{t}}{\rho}\right),
\end{aligned}
$$

where (6) is obtained from the integral identity [27]

$$
\begin{aligned}
\int_{0}^{\infty} & \log (1+t) e^{-\mu t} t^{n-1} d t= \\
& (n-1) ! e^{\mu} \sum_{i=1}^{n} \frac{\Gamma(i-n, \mu)}{\mu^{i}}, \quad n=1,2, \cdots .
\end{aligned}
$$

Note that in (6) and (7) $\Gamma(a, x)=\int_{x}^{\infty} t^{a-1} e^{-t} d t$ is the incomplete gamma function [28, p.260]. Moreover, recall the results in [2] and [29] that the theoretical ergodic capacity of an open-loop MIMO system under $N_{r} \geq N_{t}$ is

$$
\begin{aligned}
C_{\mathrm{opt}} & =\mathbb{E}\left[\log \operatorname{det}\left(\mathbf{I}_{N_{r}}+\frac{\rho}{N_{t}} \mathbf{H H}^{H}\right)\right] \\
& =e^{N_{t} / \rho} \sum_{i=0}^{N_{t}-1} \sum_{j=0}^{i} \sum_{l=0}^{2 j}\left\{\frac{(-1)^{l}(2 j) !\left(N_{r}-N_{t}+l\right) !}{2^{2 i-l} j ! l !\left(N_{r}-N_{t}+j\right) !} .\right. \\
& \left.\left(\begin{array}{c}
2 i-2 j \\
i-j
\end{array}\right)\left(\begin{array}{c}
2 j+2 N_{r}-2 N_{t} \\
2 j-l
\end{array}\right) \sum_{r=0}^{N_{r}-N_{t}+l} E_{r+1}\left(\frac{N_{t}}{\rho}\right)\right\},
\end{aligned}
$$

where $E_{r}(x)=\int_{1}^{\infty} e^{-x t} t^{-r} d t$ is the exponential integral function of order $r$ [28, p.228]. By comparing (6) and (9) under the same power constraint as well as the same number of transmit and receive antennas, we define the "efficiency" of the zero-forcing receiver as

$$
\eta=\frac{C_{\mathrm{zf}}}{C_{\mathrm{opt}}}
$$

to gauge the performance of the zero-forcing receiver in extracting the multiplexing gain of the MIMO channel.

Figure 1 plots the efficiency of the zero-forcing receiver for different numbers of transmit and receive antennas with various values of $\rho$. It is observed that the zero-forcing receiver can achieve a larger portion of the theoretical ergodic capacity of a single-user MIMO channel under the following two conditions. First, operating the zero-forcing receiver at the high SNR regime, say $\rho=30 \mathrm{~dB}$, can alleviate the noise enhancement effect and considerably improve performance. Second, by comparing the case $N_{t}=N_{r}=2$ to $N_{t}=2$ and $N_{r}=4$ (or $N_{t}=N_{r}=3$ to $N_{t}=3$ and $N_{r}=4$ ), it is found that adding more receive antennas can significantly improve the efficiency of the zero-forcing receiver to deliver multiplexing gain because the additional receive antennas provide necessary diversity for the zero-forcing receiver to reduce the noise enhancement effect.

\section{Motivation}

Based on the observations from Fig. 1, two important effects resulting from the scheduling technique can essentially improve the maximum achievable throughput performance of the zero-forcing receiver in the multiuser MIMO system. First, the multiuser selection diversity extracted by a proper scheduling algorithm can prevent the user from being served under ill channel conditions, thereby providing an intrinsically high SNR environment. Second, taking the view from the whole multiuser MIMO system, one can find that the scheduling algorithm can virtually increase the number of antennas at the receiving end to the sum of all the users' receive antennas. Therefore, while the low efficiency of the zero-forcing receiver with simple independent decoding may disqualify its use from the viewpoint of the single-user MIMO system, a practical and interesting question arises: given an underlying scheduling system such as IS-856, how well can the multiuser diversity be utilized to mitigate the adverse noise enhancement effect of the zero-forcing receiver? Towards this end, some simulation results and asymptotic analysis (as to the limit of the number of users approaching infinity) have been provided in [20] 


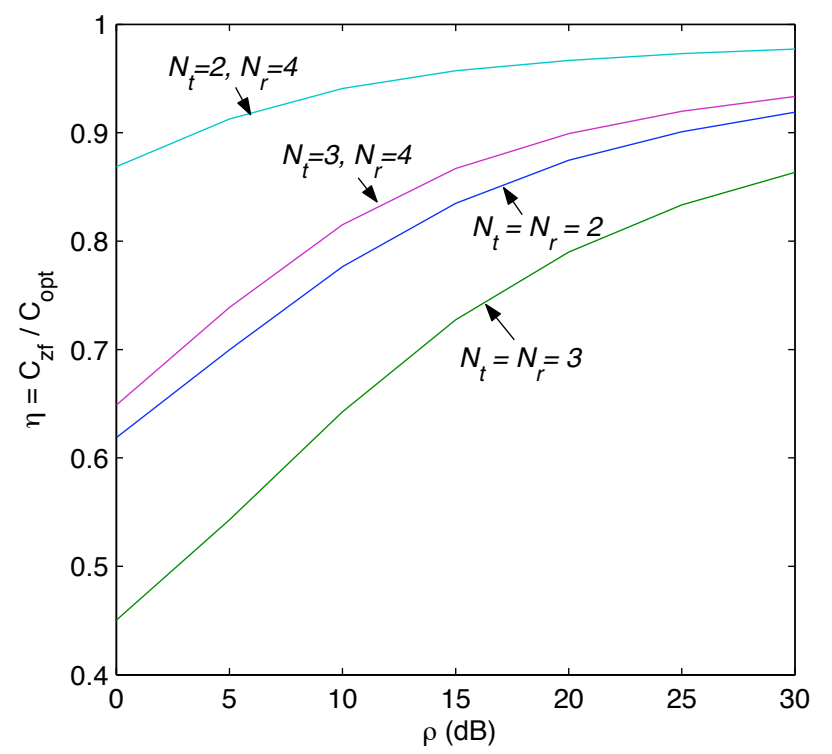

Fig. 1. Efficiency of the zero-forcing receiver in a single-user MIMO system. Here, the efficiency is defined as the maximum achievable throughput of the zero-forcing receiver divided by the theoretical ergodic capacity of an openloop MIMO system under the same power constraint as well as the same number of transmit and receive antennas.

and [21], respectively. In the following, we will introduce an analytical framework, applicable to an arbitrary finite number of users and antennas as well as a variety of scheduling algorithms, to evaluate the performance of multiuser MIMO systems.

\section{Problem Formulation}

Now we shift our focus from the single-user MIMO system to the multiuser MIMO system. In this section, we describe the system model for the multiuser MIMO downlink, explain the feedback schemes as well as scheduling policies and define the performance metric.

\section{A. The Multiuser MIMO Downlink System}

Consider a multiuser MIMO system where the base station with $N_{t}$ transmit antennas serves $K$ downlink users, each of which is equipped with $N_{r}$ receive antennas. For the purpose of decoupling the benefits of the multiuser scheduling from the extra physical receive antennas, we shall restrict our analysis to the case $N_{t}=N_{r}=N$ in the remaining part of the paper. The channel between the base station and user $k(k=1, \cdots, K)$ is represented by the channel matrix $\mathbf{G}_{k}=\sqrt{g_{k}} \mathbf{H}_{k}$ as defined in (1). We focus our analysis on the homogeneous scenario, i.e. $g_{k}=g$ for all $k$, and extend the results to the case of heterogenous users in Section V. The channel variations of $\mathbf{H}_{k}$ are assumed to be fixed during a time slot, but independently vary from time slot to time slot. The variations of $\mathbf{H}_{k}$ across users are also assumed to be independent. Thus, from (4), the PDF of the output SNR at the $n$-th subchannel of user $k$ becomes

$$
f_{\gamma_{n}^{k}}\left(\gamma_{n}\right)=\frac{N}{\rho} e^{-N \gamma_{n} / \rho} .
$$

Therefore, $\gamma_{n}^{k}$ in (11) are i.i.d. exponentially distributed random variables with parameter $\rho / N$ for all $n \in\{1, \cdots, N\}$ and $k \in\{1, \cdots, K\}$.

To tackle the total $N K$ variates in (11), order statistics is a convenient tool to analyze the performance of the multiuser MIMO system with various scheduling algorithms. We introduce the notation concerning order statistics that will be used extensively in the remaining part of this paper. Let $X_{1}, X_{2}, \cdots, X_{L}$ be a random sample from a continuous random variable with PDF $f_{X}(x)$ and CDF of $F_{X}(x)$. Let $X_{1: L} \leq X_{2: L} \leq \cdots \leq X_{L: L}$ be the order statistics obtained by arranging the preceding sample in increasing order of magnitude. Then $X_{l: L}(1 \leq l \leq L)$ is called the $l$-th order statistic and its PDF and CDF can be respectively expressed by [25]

$$
f_{X_{l: L}}\left(x_{l}\right)=\frac{L !\left[F_{X}\left(x_{l}\right)\right]^{l-1}\left[1-F_{X}\left(x_{l}\right)\right]^{L-l} f_{X}\left(x_{l}\right)}{(l-1) !(L-l) !}
$$

and

$$
F_{X_{l: L}}\left(x_{l}\right)=\sum_{i=l}^{L}\left(\begin{array}{c}
L \\
i
\end{array}\right)\left[F_{X}\left(x_{l}\right)\right]^{i}\left[1-F_{X}\left(x_{l}\right)\right]^{L-i} .
$$

Following this notation, the subchannel output $\operatorname{SNR}\left\{\gamma_{n}^{k}\right\}_{n=1}^{N}$ for any user $k$ can be arranged to have $\gamma_{1: N}^{k} \leq \cdots \leq \gamma_{N: N}^{k}$.

\section{B. Feedback Scheme for Scheduling}

The base station is assumed to have the knowledge of channel quality experienced by each user through the instantaneous feedback from all users. We consider two feedback schemes that provide the base station with different amounts of channel quality information:

- Scalar feedback: For scalar feedback it means that only a scalar value of one subchannel output SNR is sent back from each user. In Section IV, we will analyze two extreme cases wherein the maximum or minimum value of each user's subchannel output SNR (i.e. $\gamma_{N: N}^{k}$ or $\left.\gamma_{1: N}^{k}\right)$ is fed back to the base station. With the information $\left\{\gamma_{N: N}^{k}\right\}_{k=1}^{K}$ or $\left\{\gamma_{1: N}^{k}\right\}_{k=1}^{K}$ from all users, the base station follows the TDMA scheduling protocol to select one target user to serve at each time slot. Such a scalar feedback scheduling scheme requires only a low-rate feedback channel and can be applicable to the current IS856 cellular system, where a feedback channel of $800 \mathrm{~Hz}$ is supported in the reverse link and its protocol permits the base station to serve one user at a time slot [11].

- Vector feedback: The vector feedback requires a vector of $\left\{\gamma_{n}^{k}\right\}_{n=1}^{N}$ be sent back from each user. Consequently, the base station can exploit complete knowledge of $\left\{\gamma_{n}^{k}\right\}$ for all $n$ and $k$ to achieve better system performance by performing scheduling in the spatial (antenna) domain as well [20], [26]. Unlike the scalar feedback scheduling which dedicates all transmit antennas to one user, the vector feedback scheduling allows transmit antennas to independently bear data streams for different target users so that the base station can simultaneously service multiple users at a time slot. A specific scheduling algorithm that utilizes vector feedback to perform spacetime scheduling is illustrated in Section IV-C. Compared 
with the scalar feedback scheduling, the vector feedback scheduling increases the available selection order in the whole multiuser MIMO system from $K$ to $N K$. However, the vector feedback scheduling would consume more reverse link capacity due to higher feedback overheads.

\section{Performance Metric: Sum-Rate Capacity}

We use the ergodic sum-rate capacity to evaluate the performance of the multiuser MIMO system. Represent $\left\{\tilde{\gamma}_{n}\right\}_{n=1}^{N}$ as the effective output SNR for the data streams transmitting from the base station to the target user(s) after applying the scheduling technique. Also, let $\tilde{\gamma}_{1: N} \leq \cdots \leq \tilde{\gamma}_{N: N}$ be the ordered version of $\left\{\tilde{\gamma}_{n}\right\}_{n=1}^{N}$ and $f_{\tilde{\gamma}_{n: N}}\left(\gamma_{n}\right)$ denote the PDF of $\tilde{\gamma}_{n: N}$. Then, the sum-rate capacity of the multiuser MIMO system employing the zero-forcing receiver and scheduling technique can be expressed by

$$
\begin{aligned}
\tilde{C}_{\text {zf }} & =\sum_{n=1}^{N} \mathbb{E}\left[\log \left(1+\tilde{\gamma}_{n: N}\right)\right] \\
& =\sum_{n=1}^{N} \int_{0}^{\infty} \log \left(1+\gamma_{n}\right) f_{\tilde{\gamma}_{n: N}}\left(\gamma_{n}\right) d \gamma_{n} .
\end{aligned}
$$

\section{Performance Analysis for the Multiuser MIMO SYSTEM WITH SCHEDULING}

This section analyzes the performance of the multiuser MIMO system with scheduling. We first consider two scalar feedback scheduling policies that select the target user based on the extreme order statistic of $\gamma_{N: N}^{k}$ or $\gamma_{1: N}^{k}$. Then, we investigate one vector feedback scheduling strategy that can fully exploit the selection diversity order provided in the whole multiuser MIMO system.

\section{A. Max-Max Scheduling}

With the information of $\left\{\gamma_{N: N}^{k}\right\}_{k=1}^{K}$ from all users, the base station chooses the target user according to

$$
k^{*}=\arg \max _{k} \gamma_{N: N}^{k} \text {. }
$$

We call the scheduling policy of (15) the max-max scheduling in this paper. Obviously, once the target user $k^{*}$ is determined, we have

$$
\tilde{\gamma}_{n: N}^{\mathrm{ma}}=\gamma_{n: N}^{k^{*}} \quad \text { for } n=1, \cdots, N
$$

where the superscript $m a$ denotes the max-max scheduling. Recall from (11) that $\left\{\gamma_{n}^{k}\right\}$ are i.i.d. random variables across $n$ and $k$. Therefore, $\tilde{\gamma}_{N: N}^{\mathrm{ma}}$ (the highest order of $\tilde{\gamma}_{n: N}^{\mathrm{ma}}$ ) in (16) can be equivalently generated by selecting the largest one from the total $K N$ i.i.d. random variables given in (11). Representing $X$ as an exponentially distributed random variable with parameter $\rho / N$, it then follows that

$$
\tilde{\gamma}_{N: N}^{\mathrm{ma}}=X_{K N: K N} .
$$

Thus, applying (17) to (12) gives the PDF of $\tilde{\gamma}_{N: N}^{\mathrm{ma}}$ as

$$
f_{\tilde{\gamma}_{N: N}^{\mathrm{ma}}}\left(\gamma_{N}\right)=\frac{K N^{2}}{\rho} e^{-N \gamma_{N} / \rho}\left(1-e^{-N \gamma_{N} / \rho}\right)^{K N-1}
$$

Next, we proceed to derive the PDF of $\tilde{\gamma}_{n: N}^{\mathrm{ma}}$ for $n=$ $1, \cdots, N-1$ based on the obtained $f_{\tilde{\gamma}_{N: N}^{\mathrm{ma}}}\left(\gamma_{N}\right)$ in (18).

Let $f_{\gamma_{n: N}^{k^{*}}, \gamma_{N: N}^{k^{*}}}\left(\gamma_{n}, \gamma_{N}\right)$ denote the joint PDF of $\gamma_{n: N}^{k^{*}}$ and $\gamma_{N: N}^{k^{*}}$. Then the marginal PDF of $\tilde{\gamma}_{n: N}^{\mathrm{ma}}$ can be obtained by

$f_{\tilde{\gamma}_{n: N}^{\mathrm{ma}}}\left(\gamma_{n}\right)=\int_{\gamma_{n}}^{\infty} f_{\gamma_{n: N}^{k^{*}}, \gamma_{N: N}^{k^{*}}}\left(\gamma_{n}, \gamma_{N}\right) d \gamma_{N}, n=1, \cdots, N-1$.

Using the conditional probability property, we can write

$$
f_{\gamma_{n: N}^{k^{*}}, \gamma_{N: N}^{k^{*}}}\left(\gamma_{n}, \gamma_{N}\right)=f_{\gamma_{n: N}^{k^{*}} \mid \gamma_{N: N}^{k^{*}}}\left(\gamma_{n} \mid \gamma_{N}\right) f_{\gamma_{N: N}^{k^{*}}}\left(\gamma_{N}\right) \text {, }
$$

where $f_{\gamma_{N: N}^{k^{*}}}\left(\gamma_{N}\right)=f_{\tilde{\gamma}_{N: N}^{\operatorname{ma}}}\left(\gamma_{N}\right)$ is given in (18), and the conditional PDF $f_{\gamma_{n: N}^{k^{*}} \mid \gamma_{N: N}^{k^{*}}}\left(\gamma_{n} \mid \gamma_{N}\right)$ can be obtained with the help of the following lemma.

Lemma 1 [25, Theorem 2.4.2]: Let $X_{1: L} \leq \cdots \leq X_{L: L}$ be the order statistics of a sample of size $L$ from a random variable with PDF $f_{X}(x)$ and $\operatorname{CDF} F_{X}(x)$. Given that $X_{j: L}=$ $x_{j}$, the conditional PDF of $X_{i: L}$ for $j>i$ is

$$
\begin{gathered}
f_{X_{i: L}}\left(x_{i} \mid X_{j: L}=x_{j}\right)=\frac{(j-1) !}{(i-1) !(j-i-1) !}\left\{\frac{F_{X}\left(x_{i}\right)}{F_{X}\left(x_{j}\right)}\right\}^{i-1} \\
\cdot\left\{\frac{F_{X}\left(x_{j}\right)-F_{X}\left(x_{i}\right)}{F_{X}\left(x_{j}\right)}\right\}^{j-i-1} \frac{f_{X}\left(x_{i}\right)}{F_{X}\left(x_{j}\right)}, \quad x_{i} \leq x_{j}
\end{gathered}
$$

By substituting (21) into (20) and using the binomial expansion $(1-x)^{n}=\sum_{i=0}^{n}\left(\begin{array}{c}n \\ i\end{array}\right)(-1)^{i} x^{i}$, (19) can be expressed as

$$
\begin{aligned}
& f_{\tilde{\gamma}_{n: N}^{\operatorname{ma}}}\left(\gamma_{n}\right)=\frac{K N^{2}(N-1) !}{\rho(n-1) !(N-n-1) !} \sum_{i_{1}=0}^{n-1}\left(\begin{array}{c}
n-1 \\
i_{1}
\end{array}\right) \\
& \cdot \sum_{i_{2}=0}^{N(K-1)}\left(\begin{array}{c}
N(K-1) \\
i_{2}
\end{array}\right) e^{-\left(i_{1}+i_{2}+1+N-n\right) N \gamma_{n} / \rho} \\
& \cdot \sum_{i_{3}=0}^{N-n-1}\left(\begin{array}{c}
N-n-1 \\
i_{3}
\end{array}\right) \frac{(-1)^{i_{1}+i_{2}+i_{3}}}{i_{2}+i_{3}+1} .
\end{aligned}
$$

As a result, from (18) and (22), we have all PDFs of $\tilde{\gamma}_{n: N}^{\mathrm{ma}}$ for $n=1, \cdots, N$. In the following, we use these PDFs to derive $\tilde{C}_{\text {zf }}$ subject to the max-max scheduling. Applying (18) and (22) to (14), we obtain the sum-rate capacity of the multiuser MIMO system with the zero-forcing receiver and max-max scheduling as follows:

$$
\tilde{C}_{\mathrm{zf}}^{\mathrm{ma}}=C_{\tilde{\gamma}_{N: N}^{\mathrm{ma}}}+\sum_{n=1}^{N-1} C_{\tilde{\gamma}_{n: N}^{\mathrm{ma}}}
$$

where

$$
C_{\tilde{\gamma}_{N: N}^{\mathrm{ma}}}=\frac{K N^{2}}{\rho} \sum_{i=0}^{K N-1}(-1)^{i}\left(\begin{array}{c}
K N-1 \\
i
\end{array}\right) h\left(\frac{N(i+1)}{\rho}\right)
$$

and

$$
\begin{aligned}
& C_{\tilde{\gamma}_{n: N}^{\mathrm{ma}}}=\frac{K N^{2}(N-1) !}{\rho(n-1) !(N-n-1) !} \sum_{i_{1}=0}^{n-1}\left(\begin{array}{c}
n-1 \\
i_{1}
\end{array}\right) \\
& \cdot \sum_{i_{2}=0}^{N(K-1)}\left(\begin{array}{c}
N(K-1) \\
i_{2}
\end{array}\right) h\left(\frac{\left(i_{1}+i_{2}+1+N-n\right) N}{\rho}\right) \\
& \cdot \sum_{i_{3}=0}^{N-n-1}\left(\begin{array}{c}
N-n-1 \\
i_{3}
\end{array}\right) \frac{(-1)^{i_{1}+i_{2}+i_{3}}}{i_{2}+i_{3}+1}
\end{aligned}
$$


for $n=1, \cdots, N-1$. Note that $h(\cdot)$ in (24) and (25) is defined as

$$
h(x) \triangleq \int_{0}^{\infty} e^{-x t} \log (1+t) d t=\frac{e^{x} E_{1}(x)}{x}
$$

where $E_{1}(x)$ is the exponential integer function of the first order defined in (9).

\section{B. Max-Min Scheduling}

Now we consider another scalar feedback scheduling wherein the base station arranges transmissions according to $\left\{\gamma_{1: N}^{k}\right\}_{k=1}^{K}$. Note that $\gamma_{1: N}^{k}$ represents the minimum output SNR among $N$ antennas for user $k$. During each time slot, the base station selects the target user according to the following max-min scheduling policy:

$$
k^{*}=\arg \max _{k} \gamma_{1: N}^{k}
$$

Similar to (16), once the target user $k^{*}$ is selected, we have

$$
\tilde{\gamma}_{n: N}^{\mathrm{mi}}=\gamma_{n: N}^{k^{*}} \quad \text { for } n=1, \cdots, N
$$

where the superscript $m i$ denotes the max-min scheduling. From (11) and (12), it is easy to find that the feedback information $\gamma_{1: N}^{k}$ in (27) has an exponential distribution with parameter $\rho / N^{2}$. Consequently, using the scheduling rule of (27) to select the maximum from $K$ i.i.d. $\left\{\gamma_{1: N}^{k}\right\}_{k=1}^{K}$ results in

$$
\tilde{\gamma}_{1: N}^{\mathrm{mi}}=X_{K: K},
$$

where $X$ is an exponentially distributed random variable with parameter $\rho / N^{2}$. Applying (12) to (29), we obtain the PDF of $\tilde{\gamma}_{1: N}^{\mathrm{mi}}$ as follows:

$$
f_{\tilde{\gamma}_{1: N}^{\mathrm{mi}}}\left(\gamma_{1}\right)=\frac{K N^{2}}{\rho} \sum_{i=0}^{K-1}(-1)^{i}\left(\begin{array}{c}
K-1 \\
i
\end{array}\right) e^{-N^{2}(i+1) \gamma_{1} / \rho} .
$$

Next, we introduce Lemma 2, which gives the conditional distribution of a higher order statistic conditioned on a lower order statistic, to derive $f_{\tilde{\gamma}_{n-N}^{\mathrm{mi}}}\left(\gamma_{n}\right)$ for $n=2, \cdots, N$.

Lemma 2 [25, Theorem 2.4.1] : Let $X_{1: L} \leq \cdots \leq X_{L: L}$ be the order statistics of a sample of size $L$ from a random variable with PDF $f_{X}(x)$ and $\mathrm{CDF} F_{X}(x)$. Given that $X_{i: L}=$ $x_{i}$, the conditional PDF of $X_{j: L}$ for $i<j$ is

$$
\begin{aligned}
& f_{X_{j: L}}\left(x_{j} \mid X_{i: L}=x_{i}\right)=\frac{(L-i) !}{(n-j) !(j-i-1) !}\left\{\frac{f_{X}\left(x_{j}\right)}{1-F_{X}\left(x_{i}\right)}\right\} \\
& \cdot\left\{\frac{F_{X}\left(x_{j}\right)-F_{X}\left(x_{i}\right)}{1-F_{X}\left(x_{i}\right)}\right\}^{j-i-1}\left\{\frac{1-F_{X}\left(x_{j}\right)}{1-F_{X}\left(x_{i}\right)}\right\}^{i-j}, \quad x_{i} \leq x_{j}
\end{aligned}
$$

With $f_{\tilde{\gamma}_{1: N}^{\mathrm{mi}}}\left(\gamma_{1}\right)$ in (30), one can utilize Lemma 2 to derive $f_{\tilde{\gamma}_{n: N}^{\mathrm{mi}}}\left(\gamma_{n}\right)$ for $n=2, \cdots, N$ as we obtain $f_{\tilde{\gamma}_{n: N}^{\mathrm{ma}}}\left(\gamma_{n}\right)$ for $n=$ $1, \cdots, N-1$ in (22) by using $f_{\tilde{\gamma}_{N: N}^{m a}}\left(\gamma_{N}\right)$ and Lemma 1. Here, we adopt Lemma 3 to take advantage of the additive Markov chain property for the order statistics from the exponential distribution. The result of Lemma 3 was originally proved in [32] and has been applied in many problems involving the order statistics from an exponential distribution such as [33].

Lemma 3 [25, Theorem 4.6.1] : Let $X_{1: L} \leq \cdots \leq X_{L: L}$ be the order statistics of a random sample of size $L$ from an exponential distribution with parameter $\lambda$. Define a new set of random variables to denote the spacings between $X_{l: L}$ and $X_{l-1: L}$ as follows:

$$
\left\{\begin{array}{l}
S_{1}=X_{1: L}, \\
S_{l}=X_{l: L}-X_{l-1: L}, \quad l=2, \cdots, L
\end{array}\right.
$$

Then, $\left\{S_{l}\right\}_{l=1}^{L}$ are independent exponentially distributed random variables with parameter $\lambda /(L-l+1)$ for $1 \leq l \leq L$. Hence, the PDF of $S_{l}$ is given by

$$
f_{S_{l}}\left(s_{l}\right)=\frac{L-l+1}{\lambda} \exp \left(-\frac{L-l+1}{\lambda} s_{l}\right), \quad l=1,2, \cdots, L
$$

Since the spacings $S_{l}(2 \leq l \leq L)$ are independent of $X_{1: L}$ according to Lemma 3 , we can capture the statistical behavior of $\tilde{\gamma}_{n: N}^{\mathrm{mi}}$ for $n=2, \cdots, N$ by writing

$$
\tilde{\gamma}_{n: N}^{\mathrm{mi}}=\tilde{\gamma}_{1: N}^{\mathrm{mi}}+S_{2}+\cdots+S_{n} .
$$

Representing $\phi_{\tilde{\gamma}_{1: N}^{\mathrm{mi}}}(\omega)$ and $\phi_{S_{l}}(\omega)$ as the Laplace transform of $\tilde{\gamma}_{1: N}^{\mathrm{mi}}$ and $S_{l}$, respectively, we have

$$
f_{\tilde{\gamma}_{n: N}^{\mathrm{mi}}}\left(\gamma_{n}\right)=\mathcal{L}^{-1}\left\{\phi_{\tilde{\gamma}_{1: N}^{\mathrm{mi}}}(\omega) \prod_{l=2}^{n} \phi_{S_{l}}(\omega)\right\}, \quad n=2, \cdots, N
$$

where $\mathcal{L}^{-1}(\cdot)$ denotes the inverse Laplace transform. By using $\phi_{\tilde{\gamma}_{1: N}^{\mathrm{mi}}}(\omega)=\frac{N^{2} / \rho}{\omega+N^{2} / \rho}, \phi_{S_{l}}(\omega)=\frac{(L-l+1) / \lambda}{\omega+(L-l+1) / \lambda}$ and applying the partial fractional technique [28], (35) can be derived as

$$
\begin{gathered}
f_{\tilde{\gamma}_{n: N}^{\mathrm{mi}}}\left(\gamma_{n}\right)=\frac{K N^{2} \prod_{j=2}^{n}(N-j+1)}{\rho(n-2) !} \sum_{i_{1}=0}^{K-1} \sum_{i_{2}=2}^{n}(-1)^{n+i_{1}+i_{2}} \\
\cdot \frac{\left(\begin{array}{c}
K-1 \\
i_{1}
\end{array}\right)\left(\begin{array}{c}
n-2 \\
i_{2}-2
\end{array}\right)\left[e^{-\gamma_{n}\left(N-i_{2}+1\right) N / \rho}-e^{-\gamma_{n}\left(N+i_{1} N\right) N / \rho}\right]}{\left(N+N i_{1}+i_{2}-N-1\right)} .
\end{gathered}
$$

Finally, substituting the PDFs of $\tilde{\gamma}_{n: N}^{\mathrm{mi}}$ in (30) and (36) into (14), we obtain the sum-rate capacity when using the max-min scheduling policy as

$$
\tilde{C}_{\mathrm{zf}}^{\mathrm{mi}}=C_{\tilde{\gamma}_{1: N}^{\mathrm{mi}}}+\sum_{n=2}^{N} C_{\tilde{\gamma}_{n: N}^{\mathrm{mi}}}
$$

where

$$
C_{\tilde{\gamma}_{1: N}^{\mathrm{mi}}}=\frac{K N^{2}}{\rho} \sum_{i=0}^{K-1}(-1)^{i}\left(\begin{array}{c}
K-1 \\
i
\end{array}\right) h\left(\frac{N^{2}(i+1)}{\rho}\right)
$$

and

$$
\begin{gathered}
C_{\tilde{\gamma}_{n: N}^{\mathrm{mi}}}=\frac{K N^{2} \prod_{j=2}^{n}(N-j+1)}{\rho(n-2) !} \sum_{i_{1}=0}^{K-1} \sum_{i_{2}=2}^{n}(-1)^{n+i_{1}+i_{2}} \\
\cdot \frac{\left(\begin{array}{c}
K-1 \\
i_{1}
\end{array}\right)\left(\begin{array}{c}
n-2 \\
i_{2}-2
\end{array}\right)\left[h\left(\frac{\left(N-i_{2}+1\right) N}{\rho}\right)-h\left(\frac{\left(N+i_{1} N\right) N}{\rho}\right)\right]}{\left(N+N i_{1}+i_{2}-N-1\right)}
\end{gathered}
$$

for $n=2, \cdots, N$. We note that the analysis introduced in Sections IV-A and IV-B can be generalized to other scalar scheduling algorithm that uses the order statistic of $\gamma_{n: N}^{k}$ for $n \in\{2, \cdots, N-1\}$. 


\section{Spatially-Independent Scheduling}

Now we consider the vector feedback scheduling. As remarked in Section III-B, the ample information from each user's vector feedback enables the base station to perform scheduling across multiple antennas. The spatiallyindependent scheduling policy allows each transmit antenna to independently select its target user. Specifically, for the $n$-th (unordered) transmit antenna, the target user $k_{n}^{*}$ associated with the $n$-th antenna is determined according to [20]

$$
k_{n}^{*}=\arg \max _{k} \gamma_{n}^{k},
$$

where $\gamma_{n}^{k}$ is the output SNR at the $n$-th receive antenna of user $k$. We take $N=2$ and $K=3$ as an example to illustrate the selection strategy of the spatially-independent scheduling. Assume at a certain time slot the output SNR at the receive antennas of user 1,2 and 3 are $[2.3,3.5],[3.4,1.4]$ and $[3.6,1.6]$, respectively. When the max-max scheduling is used, user 3 is selected and the effective SNR sent through the multiuser MIMO system is $[3.6,1.6]$. On the other hand, using the max-min scheduling will choose user 1 and thereby yields the effective SNR of $[2.3,3.5]$. When the spatially-independent scheduling is used, the base station will simultaneously send data to users 3 and 1 through its first and second antenna, respectively. Thus, the spatially-independent scheduling results in the effective SNR of $[3.6,3.5]$ in the multiuser MIMO system.

With the effect of the spatially-independent scheduling by (40), the output SNR associated with the $n$-th antenna becomes

$$
\tilde{\gamma}_{n}^{\mathrm{si}}=X_{K: K}, \quad n=1, \cdots, N
$$

where $X$ is an exponentially distributed random variable with parameter $\rho / N$ and the superscript si denotes the spatiallyindependent scheduling. From (41), the PDF of $\tilde{\gamma}_{n}^{\text {si }}$ can be attained by invoking (12) as

$$
f_{\tilde{\gamma}_{n}^{\text {sis }}}\left(\gamma_{n}\right)=\frac{K N}{\rho}\left(1-e^{-N \gamma_{n} / \rho}\right)^{K-1} e^{-N \gamma_{n} / \rho} .
$$

Since $\left\{\tilde{\gamma}_{n}^{\text {si }}\right\}$ in (42) are i.i.d. with respect to $n$, the resulting sum-rate capacity subject to the spatially-independent scheduling can be calculated by

$$
\begin{aligned}
\tilde{C}_{\mathrm{zf}}^{\mathrm{si}} & =N \int_{0}^{\infty} \log \left(1+\gamma_{n}\right) f_{\tilde{\gamma}_{n}^{\mathrm{si}}}\left(\gamma_{n}\right) d \gamma_{n} \\
& =\frac{K N^{2}}{\rho} \sum_{i=0}^{K-1}\left(\begin{array}{c}
K-1 \\
i
\end{array}\right)(-1)^{i} h\left(\frac{(i+1) N}{\rho}\right) .
\end{aligned}
$$

Since vector feedback has provided the base station with the channel side information $\gamma_{n}^{k}$ for all $n$ and $k$, the sum-rate capacity in (43) can be further improved by using waterfilling power allocation [7] across the transmit antennas. Because the analytical treatment of the waterfilling power allocation scheme is difficult, we will provide a simulation study in Fig. 4 to compare the sum-rate capacity achieved by the equal power allocation and waterfilling power allocation.

We note that when the traditional round-robin ${ }^{1}$ (RR) scheduling policy is used in the multiuser MIMO system, the

\footnotetext{
${ }^{1}$ The round-robin scheduling policy assigns time slots to users in a circularly sequential manner.
}

multiuser diversity gain disappears and the resulting performance simply reduces to the single-user case. Under such circumstances, the sum-rate capacity of the multiuser MIMO system is equal to the maximum achievable throughput of the single-user MIMO system in (6) with $N_{t}=N_{r}=N$. That is,

$$
\tilde{C}_{\mathrm{zf}}^{\mathrm{rr}}=C_{\mathrm{zf}}=N \exp \left(\frac{N}{\rho}\right) E_{1}\left(\frac{N}{\rho}\right),
$$

where the superscript $r r$ denotes the round-robin scheduling. One can easily check that for $K=1$ the sum-rate capacity expressions (23), (37) and (43) corresponding to the max-max, max-min and spatially-independent scheduling algorithms all simplify to (44).

\section{Extension to Heterogenous Multiuser MiMO SYSTEMS}

So far we have obtained the sum-rate capacity for the homogeneous scenario wherein each user has the same average receive SNR. Now we discuss how to extend the previous results to the heterogenous case wherein each user can have different $\rho_{k}$. Since the users have near-far distribution in range of the heterogenous multiuser MIMO system, there can be differences in path loss from each user to the base station. The distance-dependent path loss, characterized by $g_{k}$ in the link model (1), can lead to different $\rho_{k}$ for different users. With the fluctuation of channel fading superimposed on $\rho_{k}$, the PDF of the instantaneous output SNR $\gamma_{n}^{k}$ in (11) for the heterogenous case becomes

$$
f_{\gamma_{n}^{k}}\left(\gamma_{n}\right)=\frac{N}{\rho_{k}} e^{-N \gamma_{n} / \rho_{k}}
$$

When the scheduling is executed purely based on the raw input of $\gamma_{n}^{k}$ in (45), the fairness issue for near-far users to compete for services could arise.

One technique for scheduling algorithms to capture the peak of channel variations while maintaining fairness among users is to use the proportional fair principle or normalization technique [30], [31]. The proportional fair principle uses the normalized SNR $\gamma_{n}^{k} / \rho_{k}$ as the metric to determine the target user. Take the max-max scheduling algorithm as an example. The selection criterion in (15) for the max-max scheduling can be modified as

$$
k^{*}=\arg \max _{k} \frac{\gamma_{N: N}^{k}}{\rho_{k}} .
$$

In (46), $\rho_{k}$ is introduced in the denominator to equalize the average SNR among different users due to the near-far effect. In practice, $\rho_{k}$ can be estimated based on long-term measurements at the user side. By doing so, any user competes for services as though the other $(K-1)$ users have the identical average SNR so that the heterogenous case virtually reduces to the homogeneous one.

Figure 2 illustrates an example of applying the modified max-max scheduling algorithm (46) to the heterogenous system with three different near-far users. As shown in Fig. 2(a), user 2 has the highest average SNR while user 3 has the lowest. With the modified max-max scheduling policy, the selected users in Fig. 2-(b) from time slots 1 to 9 are users $1,2,3,2,3,1,2,1,3$, respectively. Observing Fig. 2-(a) with 
(a): effectvie output SNR for near-far users

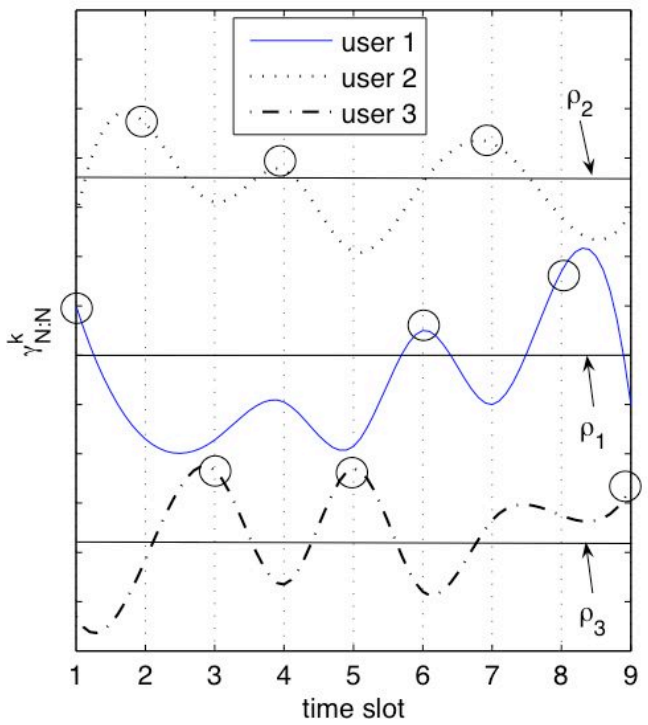

(b): normalized SNR for scheduling selection

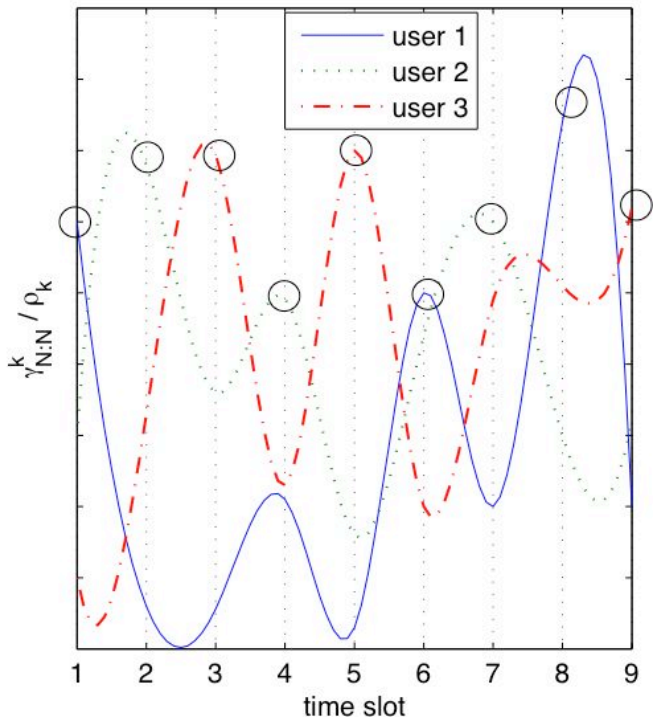

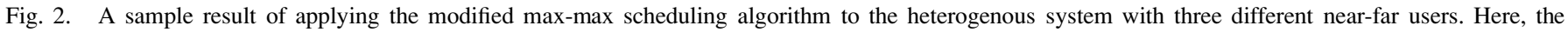
selected users from time slots 1 to 9 are users $1,2,3,2,3,1,2,1,3$, respectively.

the determined user sequence, one can find that the modified scheduling algorithm ensures that each user can be served near its peak of channel variations while maintaining fairness among users as the homogeneous scenario. Consequently, the resulting sum-rate capacity of the heterogenous system corresponds to (23) of the homogeneous system by simply replacing $\rho$ with $\rho_{k}$. The normalization technique applies to the other max-min and spatially-independent scheduling polices for heterogenous multiuser MIMO systems.

\section{NumERICAL RESUltS}

Based on the derived closed-form expressions (23), (37) and (43), this section presents some numerical results to characterize the performance gain of operating the zero-forcing receiver in the multiuser scheduling environment with reduced noise enhancement.

We first compare the CDFs of $\gamma_{n: N}$ and $\tilde{\gamma}_{n: N}$ in Fig. 3 to investigate how the various scheduling algorithms impact the effective output SNR distributions in multiuser MIMO systems. The dashed curves in the figure represent the CDFs of $\gamma_{n: N}$ while the solid curves in Fig. 3-(a) to Fig. 3(c) correspond to $\tilde{\gamma}_{n: N}$ using the max-max, max-min and spatially-independent policies, respectively. We set $N=3$, $K=16$ and $\rho=0 \mathrm{~dB}$ in this example. From Fig. 3, some observations regarding the movement of all order statistics of $\left\{\gamma_{n: N}\right\}_{n=1}^{N}$ are made as follows:

- In Fig. 3-(a), the highest order statistic of $\gamma_{3: 3}$ gains a significant boost while the lower order statistics of $\gamma_{1: 3}$ and $\gamma_{2: 3}$ only improve slightly under the max-max scheduling. In comparison, the max-min scheduling in Fig. 3-(b) enhances the magnitude of $\left\{\gamma_{n: 3}\right\}_{n=1}^{3}$ quite uniformly and the average increment of every order statistic lies between $\mathbb{E}\left[\tilde{\gamma}_{3: 3}-\gamma_{3: 3}\right]$ and $\mathbb{E}\left[\tilde{\gamma}_{2: 3}-\gamma_{2: 3}\right]$ in Fig. 3-(a).

- Observing Fig. 3-(c), one can find that $\left\{\gamma_{n: 3}\right\}_{n=1}^{3}$ are all optimally enhanced as compared with the other two fig- ures, indicating that the spatially-independent scheduling can achieve the best sum-rate capacity.

- Comparing the scalar feedback scheduling in Figs. 3-(a) and (b) with the vector feedback scheduling in Fig. 3(c), one can see that the amount of feedback information plays a crucial role of "accelerator" for scheduling techniques to effectively enhance the output SNR of all subchannels. In other words, one can make use of the scheduling technique to trade the reverse-link feedback rate for improving the downlink performance in the multiuser MIMO system.

Figure 4 characterizes the sum-rate capacity of $\tilde{C}_{\mathrm{zf}}$ under different $K$ and scheduling algorithms. The sum-rate capacity of $\tilde{C}_{\mathrm{zf}}$ is normalized to $C_{\mathrm{opt}}$, which corresponds to the sumrate capacity achieved by the optimal receiver and roundrobin scheduling in the context of multiuser MIMO systems. Fig. 4-(a) is plotted at the high SNR condition of $\rho=10$ $\mathrm{dB}$ while Fig. 4-(b) at the low SNR of $\rho=-10 \mathrm{~dB}$. It is observed that with only a small number of $K, \tilde{C}_{z \mathrm{f}}$ can be greater than $C_{\text {opt }}$ for the spatially-independent scheduling algorithm. As for the scalar feedback scheduling, $\tilde{C}_{z \mathrm{f}}$ can approach $C_{\text {opt }}$ with the increasing $K$ for both the max-max and max-min scheduling algorithms. When the zero-forcing receiver is operated at the high SNR regime, $\tilde{C}_{\text {zf }}$ can even surpass $C_{\text {opt }}$ for the max-min scheduling under moderate $K$. We also notice that the waterfilling power allocation (denoted by the curve with circles in the figure) can only add to limited capacity gain for the spatially-independent scheduling, especially when $\rho$ or $K$ is large. This is because the scheduling technique has virtually shifted the underlying communication system to a high SNR environment where the performance gap between the waterfilling power allocation and equal power allocation becomes small [7].

The performance exchange of the max-min and max-max scheduling algorithms in Fig. 4 can be explained as follows. Recall that $\tilde{C}_{\mathrm{zf}}$ is calculated by $\tilde{C}_{\mathrm{zf}}=\sum_{n=1}^{N} \mathbb{E}[\log (1+$ 
(a): max-max scheduling

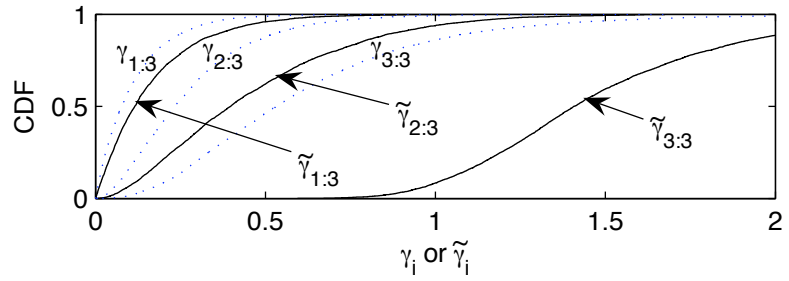

(b): max-min scheduling

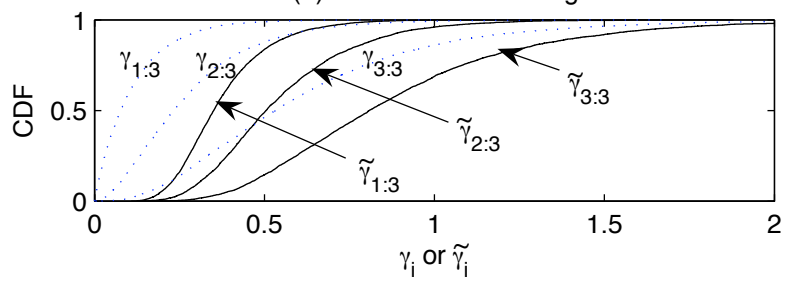

(c): spatially-independent scheduling

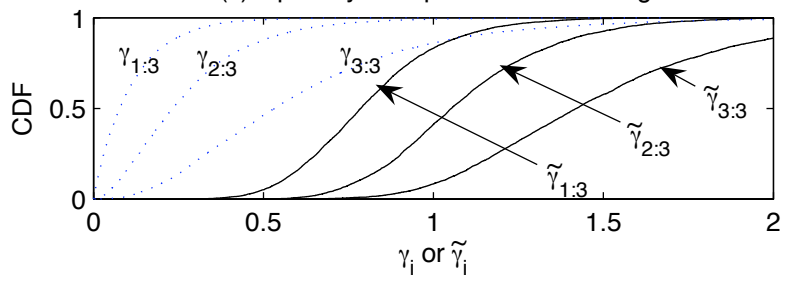

Fig. 3. The CDFs of $\left\{\tilde{\gamma}_{n: N}\right\}_{n=1}^{N}$ subject to various scheduling algorithms with $N=3, K=16$ and $\rho=0 \mathrm{~dB}$.

$\left.\left.\tilde{\gamma}_{n: N}\right)\right] \approx \sum_{n=1}^{N} \mathbb{E}\left[\tilde{\gamma}_{n: N}\right]$ at the low SNR regime. In other words, the larger the sum of $\mathbb{E}\left[\tilde{\gamma}_{n: N}\right]$, the higher the sum-rate capacity at the low SNR regime. By comparing Fig. 3-(a) and Fig. 3-(b), the significant boost of the highest order statistic $\gamma_{N: N}$ in Fig. 3-(a) thus leads to a higher resulting capacity for the max-max scheduling at the low SNR regime. On the other hand, $\tilde{C}_{\text {zf }}=\sum_{n=1}^{N} \mathbb{E}\left[\log \left(1+\tilde{\gamma}_{n: N}\right)\right]$ at the high SNR regime. Due to the concavity of the logarithm function, the contributed throughput from the enhancement of the highest order statistic $\tilde{\gamma}_{N: N}$ will hence be more suppressed than a lower order statistic $\tilde{\gamma}_{1: N}$. Since the max-max scheduling causes a larger spread of $\tilde{\gamma}_{n: N}$ than the max-min scheduling as shown in Fig. 3-(a) and Fig. 3-(b), the max-max scheduling achieves a lower resulting capacity at the high SNR regime.

Fig. 5 illustrates the impact of the number of antennas on the scheduling gain defined by $\tilde{C}_{\mathrm{zf}} / C_{\mathrm{zf}}$. Although the sum-rate capacity increases for all the studied scheduling algorithms as shown in Fig. 4, one can see from Fig. 5 that the scheduling gain reduces with the increasing $N$ for the scalar feedback scheduling. As compared with the vector feedback scheduling, the phenomena of diminishing scheduling gain for the scalar feedback scheduling may be attributed to the insufficient feedback information.

In the final example, we perform computer simulations to obtain the sum-rate capacity achieved by the optimal receiver $\left(\tilde{C}_{\text {opt }}\right)$ operating under the same scheduling policies considered in the paper. Note that the maximum achievable throughput of the optimal receiver in (8) for $N_{t}=N_{r}=N$ can be expressed as [2]

$$
C_{\mathrm{opt}}=\sum_{n=1}^{N} \mathbb{E}\left[\log \left(1+\frac{\rho}{N} \lambda_{n}\right)\right],
$$
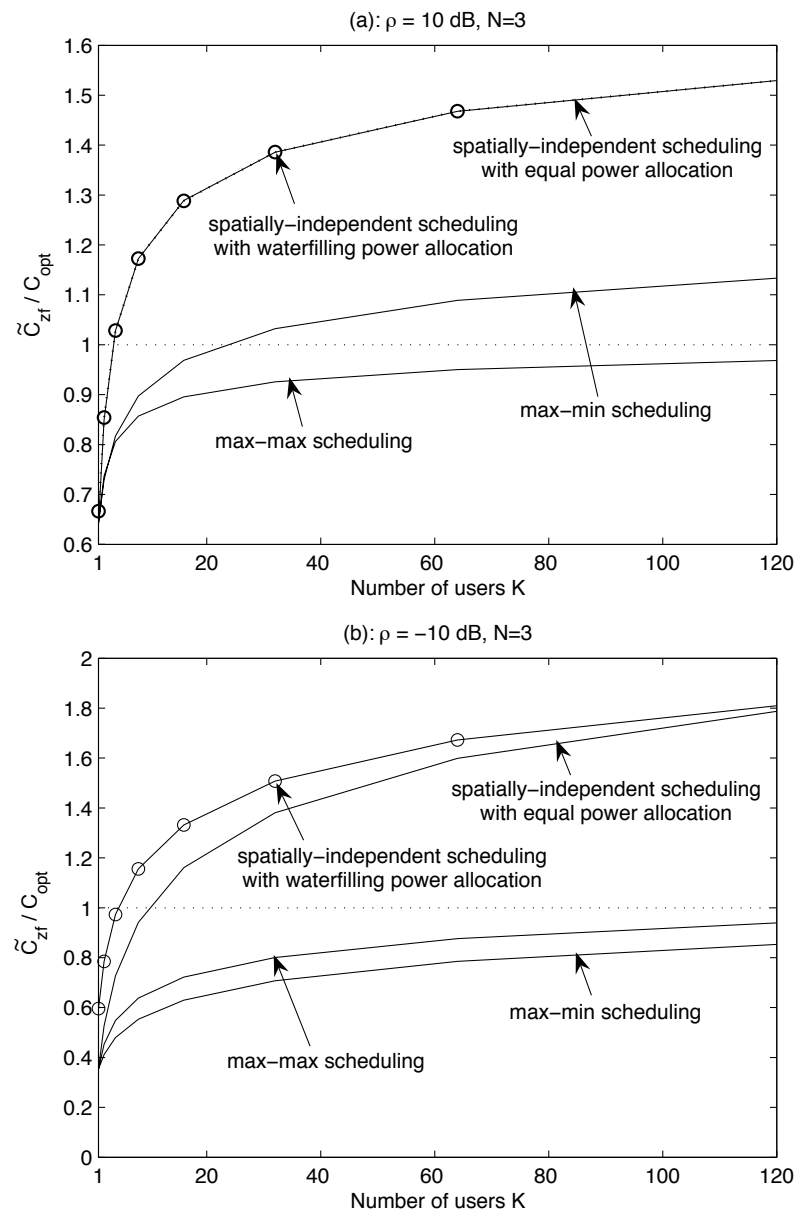

Fig. 4. The sum-rate capacity of $\tilde{C}_{\mathrm{zf}}$ under different scheduling algorithms and $K$. The curves with circles represent the spatially-independent scheduling with waterfilling power allocation.

where $\lambda_{n}$ are the eigenvalues of $\mathbf{H} \mathbf{H}^{H}$. Comparing (47) with $C_{\mathrm{zf}}=\sum_{n=1}^{N} \mathbb{E}\left[\log \left(1+\gamma_{n}\right)\right]$ in (5), the instantaneous subchannel output SNR for the optimal receiver can be represented by $\lambda_{n} \rho / N$. Therefore, by simply replacing $\gamma_{n}$ in (15), (27) and (40) with $\lambda_{n} \rho / N$, we can obtain $\tilde{C}_{\text {opt }}$ for the max-max, max-min and spatially-independent scheduling algorithms via computer simulations. Fig. 6 compares $\tilde{C}_{\mathrm{zf}}$ and $\tilde{C}_{\text {opt }}$ under the same scheduling algorithms. It is shown that the performance gap between $\tilde{C}_{\mathrm{zf}}$ and $\tilde{C}_{\mathrm{opt}}$ gradually reduces with increasing $K$ for all the scheduling policies. Since the selected users are more likely to be served at a higher SNR condition due to scheduling, the higher SNR operating environment would hence reduce the performance gap between the zero-forcing receiver and optimal receiver in extracting the multiplexing gain of the MIMO channel. This example implies a nice cross-layer cooperation synergy: since the scheduling technique has created an underlying high SNR environment for the multiuser MIMO system, one can possibly take advantage of this strength to design a MIMO receiver with lower implementation complexity without sacrificing performance too much.

\section{CONCLUSIONS}

A performance analysis of the low-cost and effective transmission strategy that employs the simple spatial multiplexing 


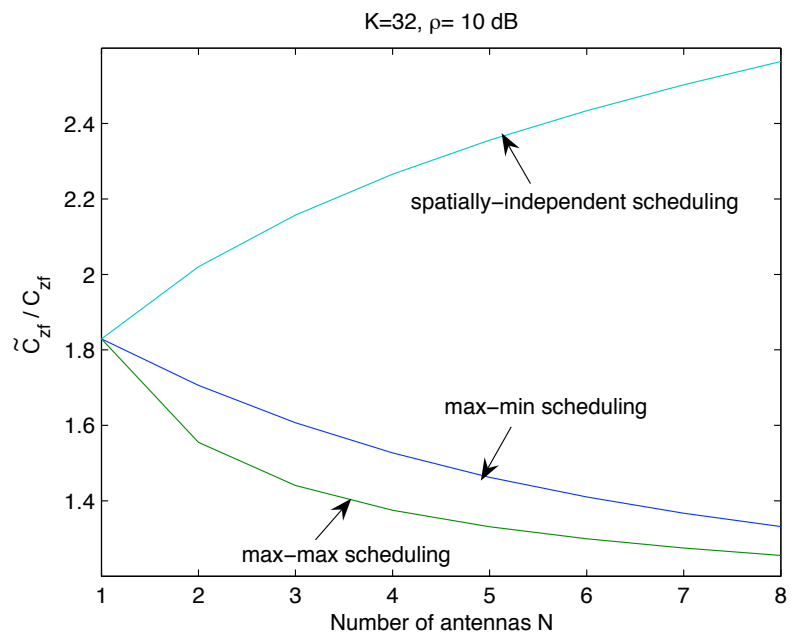

Fig. 5. Impact of the number of antennas on the scheduling gain.

at the transmitter and zero-forcing processing at the receiver in multiuser MIMO scheduling systems has been presented. By incorporating the mathematical tool of order statistics, we derive the PDFs of effective subchannel output SNRs for a variety of scheduling algorithms. These expressions are the starting point to derive the closed-form formulas, which hold for an arbitrary finite number of users and antennas, for the sum-rate capacity of the multiuser MIMO system. The closed-form expressions allow efficient numerical evaluations to characterize the capacity gain of this suboptimal transmission strategy under a number of practical scheduling policies requiring scalar or vector feedback. Our analysis and results validate the elegant marriage of the zero-forcing receiver and scheduling technique as an economical approach to achieve higher data rates for next-generation wireless communications. In the future, it is worthwhile to further investigate the optimal feedback metric and the associated scheduling algorithm for the multiuser MIMO system with the constraint of limited feedback and finite user population. Finally, we also discuss the normalization technique for scheduling in the heterogeneous scenario in the paper.

\section{REFERENCES}

[1] G. J. Foschini and M. J. Gans, "On limits of wireless communications in a fading environment when using multiple antennas," Wireless Personal Commun., vol. 6, no. 3, pp. 311-335, Mar. 1998.

[2] I. Emre Telatar, "Capacity of multi-antenna Gaussian channels," European Trans. Telecomm., vol. 10, no. 6, pp. 585-595, Nov.-Dec. 1999.

[3] C. Chuah, D. Tse, J. Kahn and R. Valenzuela, "Capacity scaling in MIMO wireless systems under correlated fading," IEEE Trans. Inform. Theory, vol. 48, pp. 637-650, Mar. 2002.

[4] M. Chiani, M. Z. Win and A. Zanella, "On the capacity of spatially correlated MIMO Rayleigh-fading channels," IEEE Trans. Inform. Theory, vol. 49, pp. 2363-2371, Oct. 2003.

[5] A. Goldsmith, S. A. Jafar, N. Jindal and S. Vishwanath, "Capacity limits of MIMO channels," IEEE J. Select. Areas Commun., vol. 21, pp. 684-702, Jun. 2003.

[6] D. Gesbert, M. Shafi, D. S. Shiu, P. Smith and A. Naguib, "From theory to practice: An overview of MIMO space-time coded wireless systems," IEEE J. Select. Areas Commun., vol. 21, pp. 281-302, Apr. 2003.

[7] A. Paulraj, R. Nabar and D. Gore, Introduction to Space-Time Wireless Communications, 1st ed., Cambridge University Press, 2003.

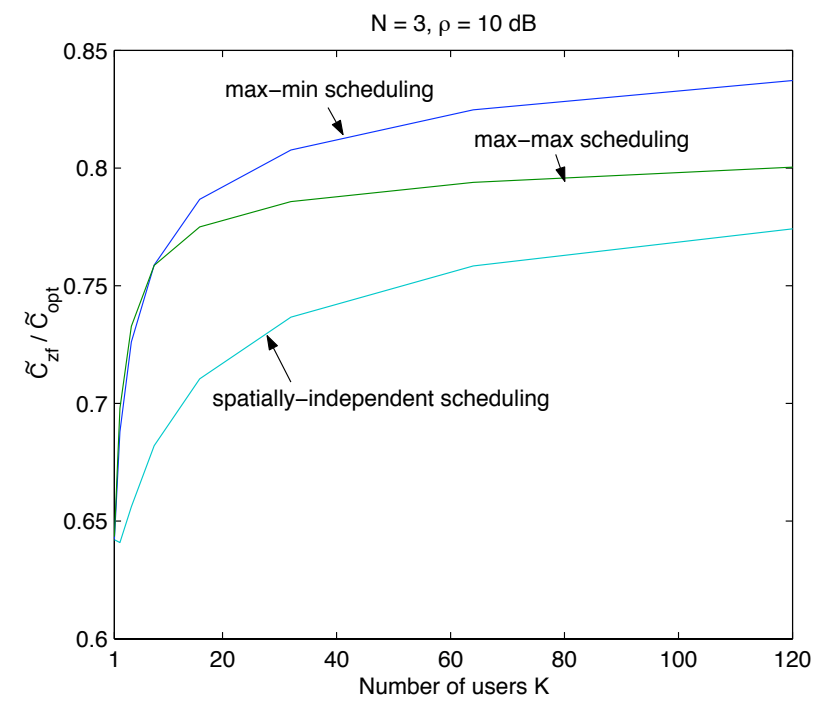

Fig. 6. Comparison of the sum-rate capacity achieved by the zero-forcing receiver $\left(\tilde{C}_{\mathrm{zf}}\right)$ and optimal receiver $\left(\tilde{C}_{\text {opt }}\right)$ operating under the same scheduling policy.

[8] G. J. Foschini, D. Chizhik, M. Gans, C. Papadias and R. A. Valenzuela, "Analysis and performance of some basic space-time architectures," IEEE J. Select. Areas Commun., vol. 21, pp. 303-320, Apr. 2003.

[9] D. Gesbert, "Robust linear MIMO receivers: a minimum error-rate approach," IEEE Trans. Signal Processing, vol. 51, pp. 2863-2871, Nov. 2003.

[10] M. Sellathurai and G. J. Foschini, "Stratified diagonal layered spacetime architectures: signal processing and information theoretic aspects," IEEE Trans. Signal Processing, vol. 51, pp. 2943-2954, Nov. 2003.

[11] TIA/EIA IS-856, "cdma2000: High rate packet data air interface specification," v.1.0.0, Dec. 2000. Available at http://www.tiaonline.org /standards/sfg/imt2k/cdma2000/TIA-EIA-IS-856-1.pdf.

[12] 3GPP TR25.950, "UTRA High Speed Downlink Packet Access," v.4.0.0, Mar. 2001. Available at http://www.3gpp.org/ftp/specs/htmlinfo/25-series.htm.

[13] S. Vishwanath, N. Jindal and A. Goldsmith, "Duality, achievable rates, and sum-rate capacity of Gaussian MIMO broadcast channels," IEEE Trans. Inform. Theory, vol. 49, pp. 2658-2668, Oct. 2003.

[14] P. Viswanath and D. Tse, "Sum capacity of the vector Gaussian broadcast channel and uplink-downlink duality," IEEE Trans. Inform. Theory, vol. 49, pp. 1912-1921, Aug. 2003.

[15] G. Caire and S. Shamai,"On the achievable throughput of a multiantenna Gaussian broadcast channel," IEEE Trans. Inform. Theory, vol. 49, pp. 1691-1706, Jul. 2003.

[16] H. Viswanathan, S. Venkatesan and H. Huang, "Downlink capacity evaluation of cellular networks with known-interference cancellation," IEEE J. Select. Areas Commun., vol. 21, no. 5, pp. 802-811, Jun. 2003.

[17] Q. Spencer, A. L. Swindlehurst and M. Haardt, "Zero-forcing methods for downlink spatial multiplexing in multiuser MIMO channels," IEEE Trans. Signal Processing, vol. 52, pp. 461-471, Feb. 2004.

[18] G. Dimic and N. D. Sidiropoulos, "On downlink beamforming with greedy user selection: performance analysis and a simple new algorithm," IEEE Trans. Signal Processing, vol. 53, pp. 3857-3868, Oct. 2005.

[19] T. Yoo and A. Goldsmith, "On the optimality of multiantenna broadcast scheduling using zero-forcing beamforming", IEEE J. Select. Areas Commun., vol. 24, no. 3, pp. 528-541, Mar. 2006.

[20] R. W. Heath, Manish Airy and A. J. Paulraj, "Multiuser diversity for MIMO wireless systems with linear receivers," Proc. Asilomar Conf. Signals, Systems, and Computers, Pacific Grove, CA, pp. 1194-1199, Nov. 2001.

[21] M. Airy, R. W. Heath and S. Shakkottai, "Multi-user diversity for the multiple antenna broadcast channel with linear receivers: asymptotic analysis," Proc. Asilomar Conf. Signals, Systems, amd Computers, pp. 886-890, Nov. 2004.

[22] M. Airy, R. W. Heath and S. Shakkottai, "Multi-user diversity for the multiple antenna broadcast channel with zero-forcing re- 
ceivers," submitted to IEEE Trans. on Wireless Commun., available at http://www.ece.utexas.edu/ rheath/papers/2006/TransWirelessComm.

[23] J. Chung, C. S. Hwang, K. Kim and Y. K. Kim, "A random beamforming technique in MIMO systems exploiting multiuser diversity," IEEE J. Select. Areas Commun., vol. 21, pp. 848-855, Jun. 2003.

[24] D. A. Gore, R. W. Heath Jr. and A. J. Paulraj, "Transmit selection in spatial multiplexing systems," IEEE Commun. Letters, vol. 6, no. 11, pp. 491-493, Nov. 2002.

[25] B. C. Arnold, N. Balakrishnan and H. N. Nagaraja, A First Course in Order Statistics, New York: Wiley, 1992.

[26] V. K. N. Lau and Y-K. Kwok, "Performance analysis of SIMO space-time scheduling with convex utility function: zero-forcing linear processing," IEEE Trans. Veh. Technol., vol. 53, pp. 339-350, Mar. 2004.

[27] M-S. Alouini and A. J. Goldsmith, "Capacity of Rayleigh fading channels under different adaptive transmission and diversity-combining techniques," IEEE Trans. on Vehicular Technology, vol. 48, pp. 11651181, Jul. 1999

[28] M. Abramowitz and I. A. Stegun, Handbook of Mathematical Functions with Formulas, Graphs, and Mathematical Tables, 9th ed. New York: Dover, 1970.

[29] H. Shin and J. H. Lee, "Capacity of multiple-antenna fading channels: spatial fading correlation, double scattering, and keyhole," IEEE Trans. Inform. Theory, vol. 49, pp. 2636-2647, Oct. 2003.

[30] J. M. Holtzman, "Asymptotic analysis of proportional fair algorithm," IEEE Symposium on PIMRC, pp. F33-F37, Oct. 2001.

[31] C. J. Chen and L. C. Wang, "Enhancing coverage and capacity for multiuser MIMO systems by utilizing scheduling," IEEE Trans. Wireless Commun., vol. 5, pp. 1148-1157, May 2006.

[32] P. V. Sukhatme, "Tests of significance for samples of the $\chi^{2}$ population with two degrees of freedom," Ann. Eugenics, vol. 8, pp. 52-56, 1937.

[33] M-S. Alouini and M. K. Simon, "An MGF-Based performance analysis of generalized selection combining over Rayleigh fading channels," IEEE Trans. Commun., vol. 48, pp. 401-415, Mar. 2000.

[34] N. Jindal and A. Goldsmith, "Dirty paper coding vs. TDMA for MIMO broadcast channels," IEEE Trans. Inform. Theory, 1783-1794, vol. 51, May 2005.

[35] M. Sharif and B. Hassibi, "A comparison of time-sharing, DPC, and beamforming for MIMO broadcast channels with many users," IEEE Trans. Commun., vol. 55, pp. 11-15, Jan. 2007.

[36] IEEE Std 802.16e-2005, "Air Interface for Fixed and Mobile Broadband Wireless Access Systems - Amendment for Physical and Medium Access Control Layers for Combined Fixed and Mobile Operation in Licensed Bands," Feb. 2006.

[37] 3GPP TR25.876, "Multiple Input Multiple Output Antennas in UTRA," v.1.8.0, Dec. 2005. Available at http://www.3gpp.org/ftp/specs/htmlinfo/25-series.htm.

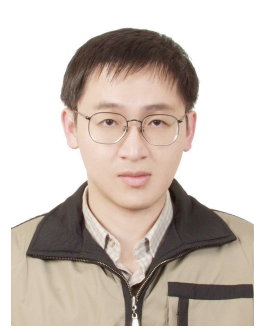

Chiung-Jang Chen was born in Kaohsiung, Taiwan, R.O.C., in May 1971. He received the B.S. degree in electronics engineering from National Chiao-Tung University (NCTU), Hsinchu, Taiwan, in 1993, the M.E. degree in electrical engineering from National Taiwan University, Taipei, Taiwan, in 1995 and the $\mathrm{Ph} . \mathrm{D}$. degree in communication engineering from NCTU, Taiwan, in 2005. He is now with Chunghwa Telecom Laboratories, Chung-Li, Taiwan. His research interests include radio network resource management, MIMO performance analysis and crosslayer optimization for high speed wireless networks.

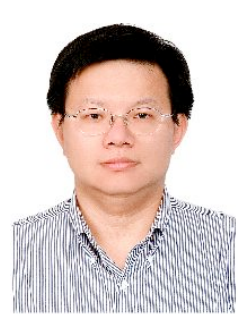

Li-Chun Wang received the B.S. degree from National Chiao Tung University, Taiwan, R.O.C. in 1986, the M.S. degree from National Taiwan University in 1988, and the Ms. Sci. and Ph. D. degrees from the Georgia Institute of Technology, Atlanta, in 1995, and 1996, respectively, all in electrical engineering.

From 1990 to 1992 , he was with the Telecommunications Laboratories of the Ministry of Transportations and Communications in Taiwan (currently the Telecom Labs of Chunghwa Telecom Co.). In 1995, he was affiliated with Bell Northern Research of Northern Telecom, Inc., Richardson, TX. From 1996 to 2000, he was a Senior Technical Staff Member in the Wireless Communications Research Department of AT\&T Labs-Research. Since August 2000, he has been an Associate Professor in the Department of Communication Engineering of National Chiao Tung University in Taiwan and become a full processor in Aug. 2005. His current research interests are in the areas of adaptive/cognitive wireless networks, radio network resource management, cross-layer optimization, and cooperative wireless communications networks.

Dr. Wang was a co-recipient (with Gordon L. Stuer and Chin-Tau Lea) of the Jack Neubauer Best Paper Award of the IEEE Vehicular Technology Society in 1997. He has published over 30 journal and 70 international conference papers and is holding three US patents. He served as an Associate Editor for the IEEE Trans. on Wireless Communications from 2001 to 2005, the guest editor of the special issue on "Mobile Computing and Networking" for IEEE Journal on Selected Areas in Communications in 2005 and the special issue on "Radio Resource Management and Protocol Engineering in Future IEEE Broadband Networks" for IEEE Wireless Communications Magazine in 2006. 